\title{
Rotation Sensors - A Brief Guide of Use of the Most Common Types
}

\author{
M. Papoutsidakis \\ Dept. of Automation Eng. Piraeus \\ University of Applied Sciences,
} Athens, Greece

\author{
D. Piromalis \\ Dept. of Automation Eng. Piraeus \\ University of Applied Sciences, \\ Athens, Greece
}

\author{
D. Tseles \\ Dept. of Automation Eng. Piraeus \\ University of Applied Sciences, \\ Athens, Greece
}

\begin{abstract}
A sensor is a device, module, or subsystem whose purpose is to observe the events or changes in its environment and send the information to other electronics, frequently a computer processor [1]. There are plenty of sensor types that are used in industry for various purposes. One type of the sensors is position sensors which are divided into Linear and Rotational sensors. This report is dedicated to the rotational sensors by specifying several different types of them. These types are Incremental rotary encoders, Absolute encoders and Inductive rotary sensors. Incremental rotary encoders measure or record length, angles, thickness and speed. They are often combined with counting, visualizing and control systems in order to achieve programmed positions, preset step shifts or to display measured values. Absolute rotary encoders are ideal to control motions and to determine position on industrial controls of operating robots and machines. Inductive rotary sensors are contactless sensors used to measure position, rotation, or speed. They work using transformer principles where current carrying coils induce a current in a metal disk.
\end{abstract}

\section{Keywords}

RVDT, RVIT, quadrate, encoder, decode, 'incoder', OEM, PCB.

\section{INTRODUCTION}

The Rotary Motion Sensor monitors angular motion precisely and easily. It is direction sensitive and it is used to collect angular displacement, angular velocity, and angular acceleration data. Typical experiments include measuring moments of inertia, torque, pendula, and Atwood's machine experiments. Sensors able to detect three-dimensional motion have been commercially available for several decades and have been used in automobiles, aircraft and ships. However, initial size, power consumption and price had prevented their mass adoption in consumer electronics. Perhaps the simplest rotational sensor is using a potentiometer as a voltage

divider where the voltage is proportional to the angle of rotation. Quadrature encoders, commonly used for measuring rotational position, provide a specific number of equally spaced pulses per revolution. A quadrature encoder is a type of incremental encoder. Incremental encoders provide relative position feedback (and can also provide speed and direction) by generating a stream of binary pulses that are proportional to the rotation of a motor's shaft.

\section{METHODS}

\subsection{Rotary Encoders}

An encoder is an electromechanical sensor of mechanical motion or position. There are two various types of encoders: [2]
1. Linear encoders (respond to motion along a path)

2. Rotary encoders (respond to rotational motion)

Rotary encoders are electromechanical devices those are used for detecting the angular position or motion of a shaft or axle by converting rotational mechanical displacements into electrical signals and processing those. They are also called shaft encoders. Rotary encoders are usually placed at the side which is perpendicular to the shaft and they are using for detecting angle, speed, length, position and acceleration in automation field. [14]

Rotary Encoders can be classified in various categories according to either their Output Signal or Sensing Technology. Classification of rotary encoders is clearly seen from the Figure 1. [3]

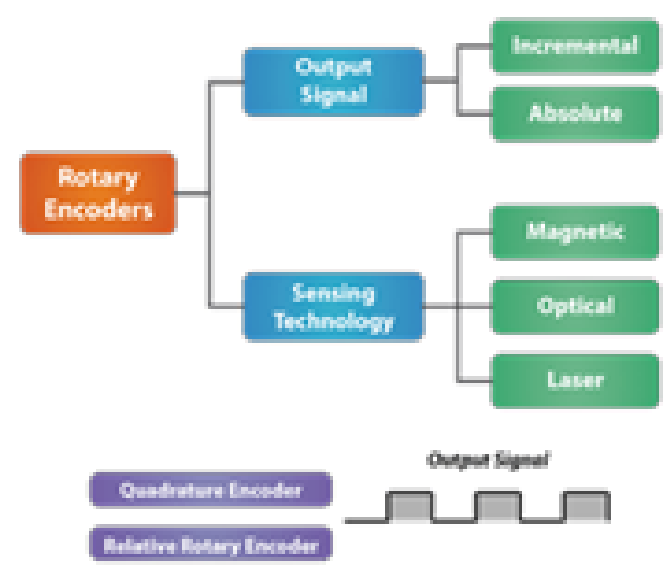

Figure 1. Classification of rotary encoders

\subsection{Incremental Rotary Encoders}

Incremental Rotary Encoders are also known as quadrature (dual-channel designs) or relative rotary encoders. This type of encoder utilizes sensors that use optical, mechanical or magnetic index counting for angular measurement. They provide speed, direction and relative position feedback by generating a series of digital square wave pulses proportional to the rotation of a motor or driven shaft.

\subsection{Features of Incremental Rotary Encoders}

Single-channel incremental encoders feature a single set of pulses, so they can only provide limited information. For instance, they can define the number of pulses in one revolution. Encoder with one set of pulses cannot indicate the direction of motion, that is why they are usually used for applications like single direction conveyor systems. Plenty of incremental encoders have the second of pulses those have 
phase difference of $90^{\circ}$. When two set of pulses are used, since they are out of phase from each other, the rotating direction can be determined from the amount of phase shift between the first and second set of pulses. So, they provide robust solutions for challenging applications. As an example, for applications that have high vibrations, single-channel encoders are not good choice, since they can count the pulse stream generated by axis dithering about a set-point as a real displacement, but double-channel encoders would be able to recognize the changes in direction and ignore the pulse stream or filter it as a noise. [4]

The set of pulses are indicated as A pulses and B pulses, respectively. The third phase signal is the light source that is indicated as phase $\mathrm{Z}$ and generated once per revolution to detect a single pulse. [5] It outputs incremental changes from a pre-defined home position. There are three additional differential signals in some incremental encoders such "/A", " $B$ " and " $Z$ ". They are inverted version of " $A$ ", " $B$ " and " $Z$ " signals and are used by controllers to ensure that there is no error during transmission. So, each pair must be equal (" $\mathrm{A}$ " = "/A", "B" = "/B" and "Z" = “/Z"). [7]

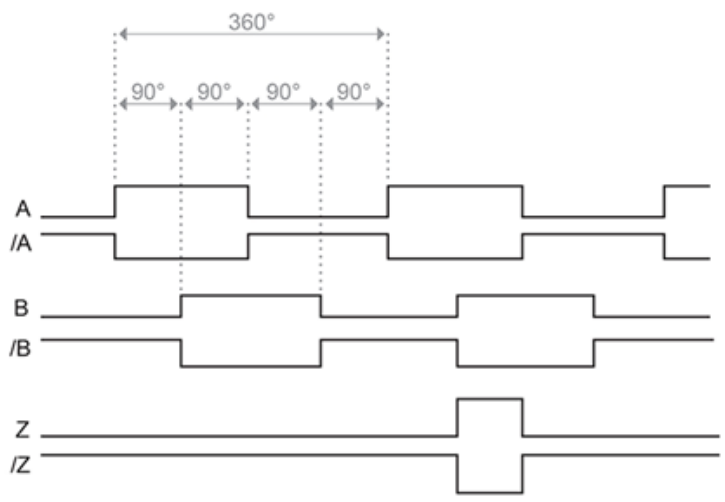

Figure 2. Incremental Pulse Diagram

Some separate electronics (typically a PLC, counter, or drive) is used to counts the number of output pulses to determine the amount of rotation based on the count. The point where the counting begins depends on the counter in the external electronics and not on the position of the encoder. To provide useful position information, the encoder position must be referenced to the device to which it is attached, generally using an index pulse. If it is needed to determine the amount of rotation from a certain input shaft position, the count in the counter should be reset at the reference position, then counter starts to count from that point. So, reference position can be changed. If higher resolution is required, it can be increased two or four times with adding additional a 4-multiplier circuit. In this case the rise and fall waveforms of phase A and phase $\mathrm{B}$ are differentiating and four times higher resolution output is obtained. [6] The distinguishing feature of the incremental encoder is that it reports an incremental change in position of the encoder to the counting electronics. [7]

\subsection{Structure and working principle of Incremental Rotary Encoders}

Incremental rotary encoders have a transparent disk revolves along with the shaft, which contains evenly spaced opaque sections and it used to determine movement. The shaft, in turn, is mechanically connected to a mechanical device that is to be instrumented so that its speed and/or position can be monitored. A light emitting diode is used to pass through the glass disk. Light that pass through between two slits is transmitted or blocked accordingly. Transmitted light is detected by a photo detector and converted to electrical currents that corresponds to each slit. So, a train of square pulses is generated as it rotates [1]. As the disk rotates faster, the pulse frequency increases accordingly. Since the relationship between pulse frequency and rotational velocity is linear, it is a simple matter to use the pulse frequency as an indication of speed. The output pulses can be transformed into speed units by measuring their frequency, and then multiplying the frequency by an appropriate scale factor.

In the Figure 3 structure of the incremental rotary encoder is illustrated.

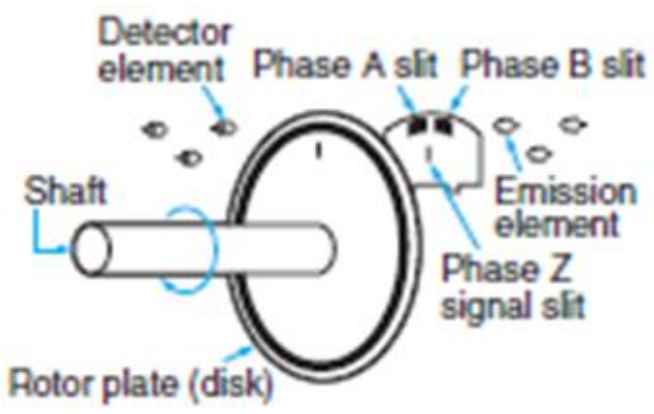

Figure 3. Structure of Incremental Rotary Encoders

After the output signal is generated it is required to determine either rotated position or rotating direction as well. For rotated position it is enough to look through any of two outputs with just counting the pulses of the signal. For determining the rotation direction as already mentioned both signals should be analyzed at the same time and there is $90^{\circ}$ phase difference between them with independent from revolution. If the rotating direction is clockwise then output $\mathrm{A}$ will be ahead of output B. In contrast, if output B leads output A, it means rotating direction is counter clockwise. [4]. In the Figure 4 how to determine rotation direction is illustrated clearly.

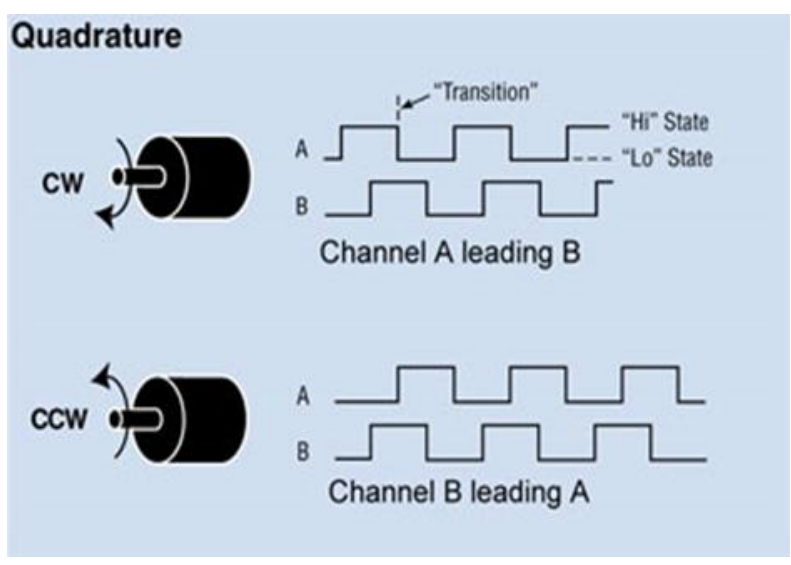

Figure 4. Determining rotating direction

For decoding in software, the A and B outputs are read by software either via an interrupt on an edge or polling and for decoding the below table is used.

\begin{tabular}{|l|l|l|}
\multicolumn{3}{|c|}{ Table 1 } \\
\hline Phase & A & B \\
\hline 1 & 0 & 0 \\
\hline 2 & 0 & 1 \\
\hline 3 & 1 & 1 \\
\hline 4 & 1 & 0 \\
\hline
\end{tabular}




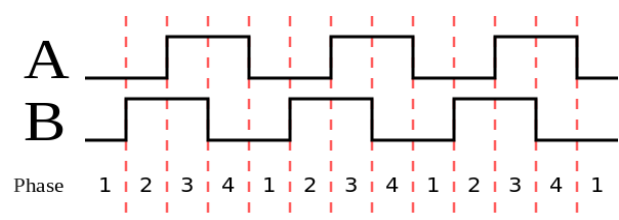

Figure 5. Two square waves in quadrature (clockwise rotation)

For instance, if the latest value was 00 , then current value is 01 and the device has rotated one half step in clockwise direction. [7]

\subsection{Applications of incremental encoders}

They can be used in positioning and motor speed feedback applications and all includes servo/light-, industrial- or heavyduty applications. An incremental encoder provides excellent speed and distance feedback. Since a few sensors are using, the systems are both simple and inexpensive. [8]

\subsection{Absolute Rotary Encoders}

Every absolute rotary encoder is used to determine the speed or position of something- the difference is how that encoder determines that movement. One of the major drawback of the incremental encoders is that the number of pulses that are counted are stored in a buffer or external counter. When the system is powered off, for instance, during temporary power outage, it does not track any incremental change output by the encoder and the count will be lost. This means that that if a

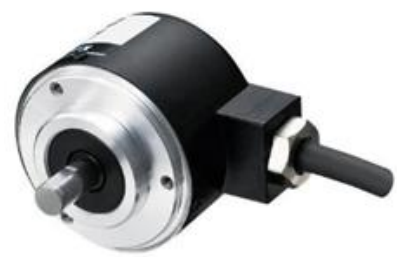

Figure 6. Absolute Rotary Encoder

Machine with an encoder has its electricity turned off each night or for maintenance, the encoder will not know its exact position when power is restored. The absolute encoder is designed to correct this problem. It is designed in such a way that the machine will always know its location. The encoder must utilize a home-detection switch to indicate correct machine position. When the home limit switch is activated, the buffer or counter is set to zero and the system knows where it is relative to fixed positional points. [4] Unlike an incremental encoder, an absolute encoder does not generate output as pulses but as digital words that identify its position as a static reference point within an absolute coordinate system. As a result, even in the event of power outage, an absolute encoder maintains record of its absolute position. Upon restart, the system can resume motion immediately, without rehoming. An absolute encoder contains components that also found in incremental encoders. They implement a photo detector and LED light source but instead of a disc with evenly spaced lines on a disc, an absolute encoder uses a disc with concentric circle patterns.

\subsection{Working principle and structure of absolute rotary encoders}

An absolute rotary encoder determines its position using a static reference point. The method is slightly different depending on whether the absolute rotary encoder is optical or magnetic, but principle is the same either way. Absolute encoder has an encoder disc which has marks or slots on a power-transmission shaft allows the system essentially to create a unique binary identifier for each point of travel and a stationary pickup. As the code disc rotates atop the fixed mask, the system periodically reads out the identifier, outputting it as a multi-bit digital word. The disc records a unique code for each shaft position. The associated controller or drive polls the encoder to capture position data that it can use directly or process into velocity information. Each position corresponds to a unique code, and even movements that occur while there is no power are recorded into accurate position values once the encoder is turned on again.

Absolute encoder outputs in a parallel rotation angle as an absolute value in $2 \mathrm{n}$ code. It therefore has one output for each output code bit, and as the resolution increases, the value of the outputs increases. Rotation position is detected by directly reading the output code. Another key feature of absolute encoders is the different output options. Encoders can't just collect feedback data; they have to send it somewhere in a language that the larger system can understand. Absolute encoder uses binary coding, which is translatable into many different protocols. If you have multiple components using the same communications bus, then it is critical that the absolute rotary encoder can communicate with the bus. [6]

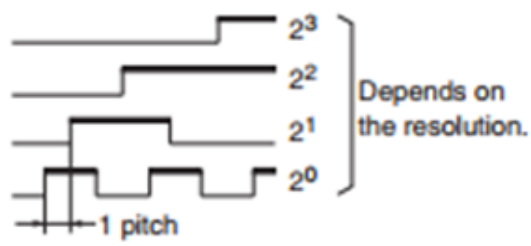

\section{Figure 7. Output waveform of the absolute encoder}

When the Encoder is incorporated into a machine, the zero position of the input revolution shaft is fixed, and rotation angle is always output as a digital value with the zero position as the coordinate origin. Data is never corrupted by noise, and returning to the zero position at startup is not necessary. Furthermore, even when code reading becomes impossible due to high speed rotation, correct data can be read when the rotation speed slows, and correct rotation data can even be read when the power is restored after a power failure or other interruption in the power supply.

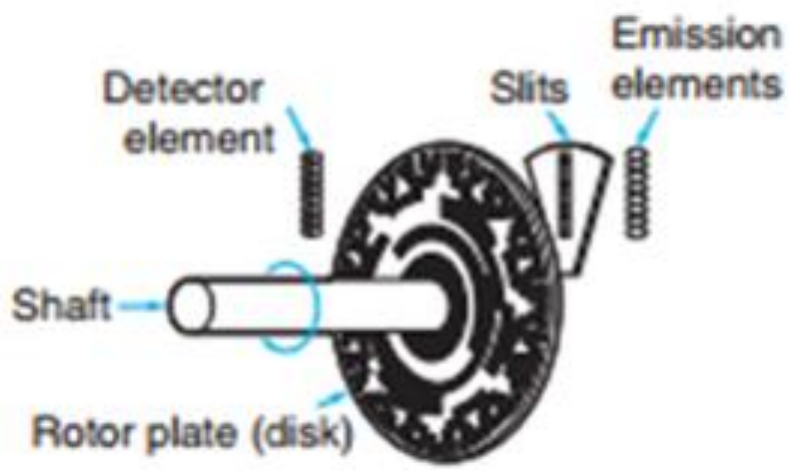

Figure 8. Structure of absolute rotary encoders 
When a disc with a pattern rotates, light passing through the slits is transmitted or blocked according to the pattern. The received light is converted to electrical currents in the detector elements, takes the form of waves, and becomes digital signals.

By having an integrated reference, an absolute rotary encoder is intrinsically able to deliver higher quality feedback:

- Higher overall resolution and orientation

- Better start up performance because of homing (or initial position) time

- Accurate motion detection along multiple axes

- Multiple output protocols for better electronics integration

- Better recovery from system or power failures

There are two versions of absolute encoders: single-turn and multi-turn encoders. Single-turn encoders measure displacement in one turn or across $360 \mathrm{deg}$. from a starting position. They divide a mechanical revolution into a certain number of measuring steps. The output repeated for every revolution. The maximum resolution of the single-turn encoder is 8192. Multi-turn encoders measure in the same fashion as single-turn, but also track the number of total revolutions of the shaft using a unique word for each position and number of revolutions. The signals are output via an SSI interface or a bus system such as CAN or Profitbus. [9]

\subsection{Advantages and disadvantages of the rotary encoder}

There are several advantages of the absolute rotary encoders: [10]

- Remembers an object's position after a power outage and offers continuous position monitoring

- Typically have speed, scaling, preset, fieldbus functions

- Allow you to determine the exact position of a machine and control over the storage of electronic data

- Single-turn and Multi-turn revolution options are available

- Absolute encoders have a resolution of up to 16 bits, or 65,536 pulses per revolution (PPR).

Usually, a disadvantage of this type of encoder is that they are expensive and hard to interface to because the number of wire connections is directly proportional to the number of resolution bits. This means that larger parallel cables are needed and either a lot of I/O on a processor, or discrete logic (or both).

In real life, absolute encoders allow more precise work applications:

- Determining multi-axis orientation for CNC machines used in parts manufacturing

- Automatically determining the height of scissor beds used in hospitals.

- Accurately positioning unique stabilizers for large vehicles like cranes or aerial lifts

- Moving automatic doors or bays without a limiting switch

- Continuing robotic movement cleanly even after a power failure

\subsection{Inductive Rotary Sensors}

Inductive sensors are usually used to measure the position or speed of the motors, especially in harsh environments. Inductive position and speed sensors are in wide variety of shapes, sizes and designs. Almost all inductive sensors work using transformer principles and they work with the phenomenon based on alternating electrical currents. This was first discovered by Michael Faraday in the 1830s when he found that a first current-carrying conductor could 'induce' a current to flow in a second conductor. Faraday's discoveries went on to deliver electric motors, dynamos and, of course, inductive sensors for position and speed measurement [11]. The inductive position sensing technology contains two main components - a Target (or rotor) and an Antenna (stationary PCB coils). The Antenna is active element, but the Target is passive one. An electrical output from the Antenna shows the rotary position of the Target relative to the Antenna. Simple proximity switches, variable inductance sensors, variable reluctance sensors, synchros, resolvers, rotary and linearly variable differential transformers (RVDTs \& LVDTs), and new generation inductive encoders can be the examples of such inductive sensors. [15]

There are several Inductive Rotary Sensors and one of them is Rotary Variable Inductance Transducer (RVIT). The RVITs are very cost-efficient, DC operated non-contact sensors (Figure 9).

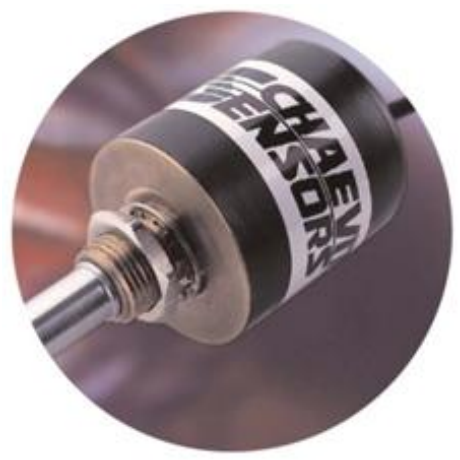

Figure 9. Rotary Variable Inductance Transducer

They can be calibrated over the full 60 or 120 degrees sensing range and offer exceptional performances. Operating from a single DC voltage, they provide either a \pm 3 VDC (RVIT 1560 ) or $4-20 \mathrm{~mA}$ (RVIT $15-120 \mathrm{i}$ ) output, over a 60 or 120 degrees angular sensing range.

\subsection{Working principle}

During operation, the conductive spoiler rotates with the transducer shaft, altering the magnetic field generated by the printed circuit coils (Figure 10). The resulting unbalance is precisely measured using a patented autoplexing circuit. This signal is then converted to a linear DC output signal that is directly proportional to the angle of the rotor shaft (Figure 11). 


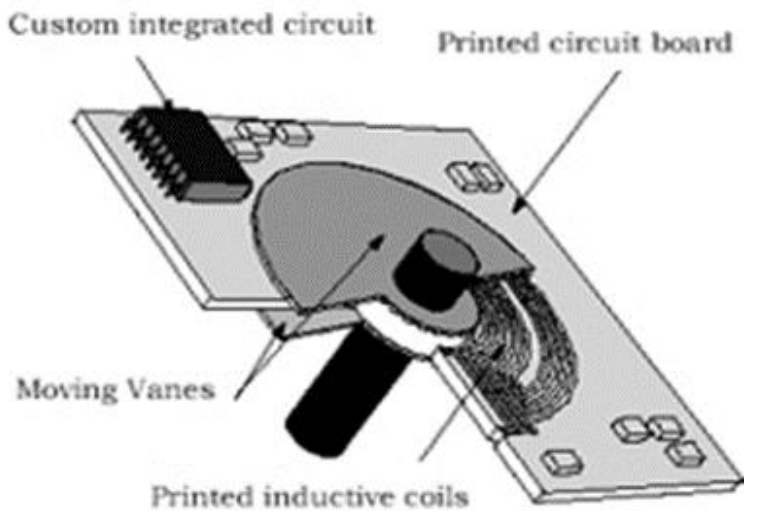

Figure 10. The construction of RVIT

The predominantly digital circuit is very resistant to environmental disturbances and is ideally compatible for use with most digital electronics. RVITs are available with a choice of standard face mounting or optional four-holes flange mounting. A shaft seal is available with flange mounting for applications where contamination is critical. RVITs offer wide operating temperature range, infinite resolution, and a virtually infinite rotational life.

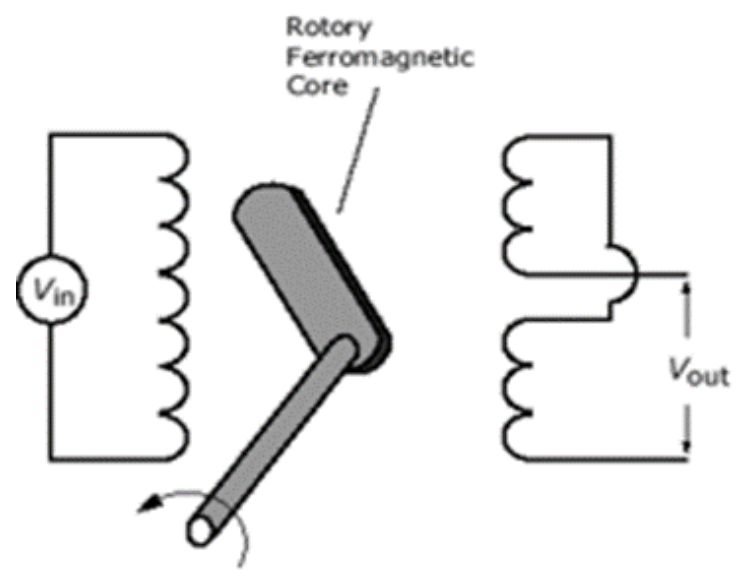

Figure 11. The working principle of RVIT

There are different types of RVITs that can be distinguished for their measuring ranges. Each of them has its own special characteristics, advantages, and disadvantages. Some of them are mentioned below:

The R60D RVIT (Rotary Variable Inductance Transducer) is a DC operated non-contact angular position sensor. Operating on a \pm 15 VDC supply, the R60D provides a smooth $\pm 7.5 \mathrm{VDC}$ output proportional to angular position over a \pm 60 degrees sensing range (Figure 12). The R60D also has improved resistance to electromagnetic disturbances and built in voltage regulation for guaranteed operation in applications where high noise environments exist. Typical applications include ball valve position feedback, throttle and position level feedback and actuation feedback [12].

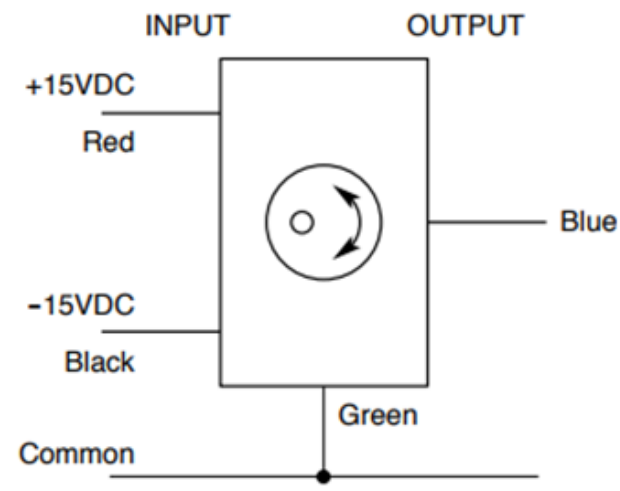

Figure 12. R60D RVIT

The RVIT-Z series is the first in the series developed from Schaevitz of flat non-contact rotary displacement sensors [12]. The unique design of the RVIT-Z combines the proprietary RVIT (Rotary Variable Inductive Transformer) technology and signal conditioning circuitry in a single PCB (Printed Circuit Board). The RVIT-Z provides an ultra-low profile, high accuracy solution for rotary and angular measurements in OEM (Original Equipment Manufacturer) applications (Figure-5). The lack of wipers, brushes, slip rings or magnetic materials eliminates wear, static friction, hysteresis and electrical noise. Measuring a fraction of the size of other rotary positioning sensors, the RVIT-Z is ideal for space critical rotary sensing applications; it is smaller, flatter and lighter than conventional rotary sensors. Capable of absolute rotary measurement over $\pm 60^{\circ}$, and extended operation up to $\pm 75^{\circ}$ (with compromised linearity), the RVIT-Z provides unsurpassed performance over an extended operating temperature range of $-40^{\circ} \mathrm{C}$ to $125^{\circ} \mathrm{C}$. For applications where remote sensing is required, the RVIT-Z can be tailored allowing the rotary sensing element to remain separated from the electronic circuitry by -up to 12 inches.

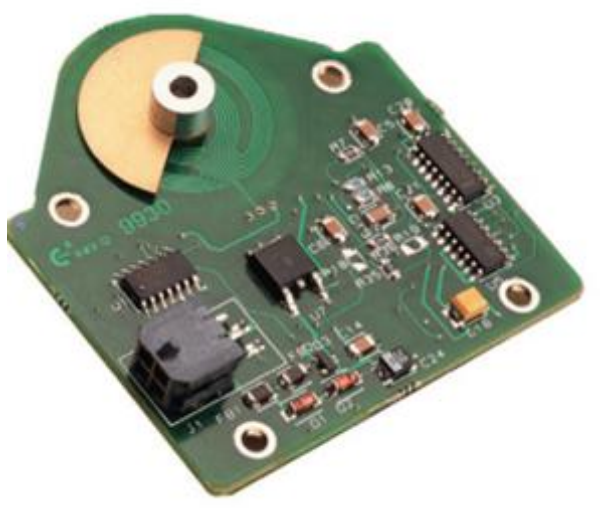

Figure 13. RVIT-Z series

The RVIT-15-60 (Figure 14) and RVIT-15-120I (Figure 15) rotary transducers are available in a variety of versions which provide a range of supply and output configurations. Calibrated outputs of \pm 3 VDC and 4-20 mA offer ideal flexibility for specialized OEM designs where unique power supply and interfacing requirements exist. Other specialized ranges, internal regulation and custom calibrated outputs are available for special order. The standard RVIT 15-60 transducer imitates a potentiometer in a way that any change in input voltage results in a proportional change in output 
voltage. Although this output is ratiometric, the RVIT-15-60 offers a considerably higher scale factor of $50 \mathrm{mV}$ per degree over an extended range of \pm 60 degrees. In addition, the noncontact design of the RVIT provides virtually infinite rotational life and extremely high accuracy of $\pm 0.25 \%$ FS. [12]

\section{RVIT-15-60}

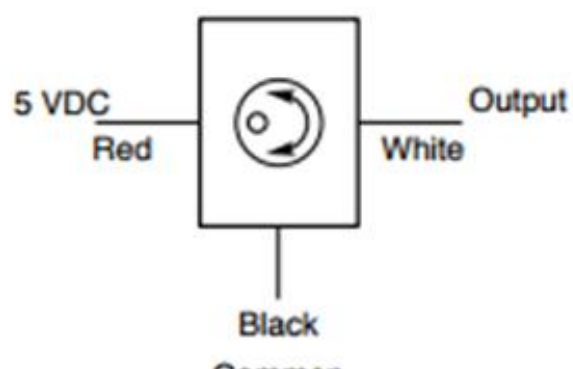

Figure 14. RVIT-15-60

\section{RVIT-15-120I}

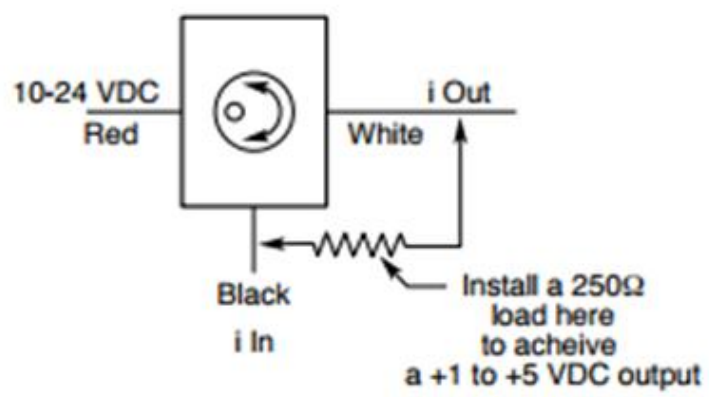

Figure 15. RVIT-15-120i

The R120LC RVIT (Rotary Variable Inductance Transducer) is a cost efficient, DC operated non-contact angular position sensor. It provides a smooth 0.5 to $4.5 \mathrm{VDC}$ output, ratiometric to the supply voltage and proportional to angular position over the 120 degrees sensing range. The R120LC utilizes non-contacting, differential inductive technology, and does not suffer the wear problems experienced by potentiometers. The R120LC proprietary design incorporates a set of printed circuit coils and a conductive spoiler to achieve superior performance and low cost. The predominantly digital circuit is very resistant to environmental disturbances and ideally suited to most industrial applications [12].

\subsection{Advantages of Inductive Rotary Sensors}

Inductive Rotary sensors have robust operation due to no moving electrical contacts, good temperature performance and resilience to dust, dirt, etc. These sensors are absolute devices which means that they need no motion at power up to determine position. Other advantages are their higher accuracy, no built-in electronics and being able to withstand harsh environments.

\subsection{Disadvantages of Inductive Rotary Sensors}

Although the inductive sensors can be used in harsh environments they are too big, bulky and costly, due to the wound transformer construction. Moreover, it is difficult for a design engineer to specify inductive sensors, and this is another reason for their relative scarcity. Each sensor often needs the associated AC generation and signal processing circuitry to be separately specified and have to be individually purchased, requiring a substantial amount of skill and knowledge of analog electronics. Since younger engineers tend to focus on digital electronics, they regard such disciplines as an unwanted 'black art' to be avoided [13]

In recent years, a new generation of inductive sensor inductive encoder has a growing reputation in the traditional and mainstream sectors. It is also called 'incoder' that is a mix of inductive and encoder. The technique used here is the same basic physics as used in traditional devices, but instead of the bulky transformer constructions and analog electronics, the new device uses printed circuit boards (PCB) and modern digital electronics. The elegant approach opens up several applications for inductive sensors such as 2D and 3D sensors, curvilinear geometries, short throw $(<1 \mathrm{~mm})$ linear devices, and high precision angle encoders, including small and large rotary encoders [13].

PCBs enables sensors to be printed onto thin flexible substrates, which can also eliminate the need for traditional cables and connectors. Similar to the previously mentioned inductive sensing techniques, the new approach also offers reliable and precision measurement in harsh working environments. Other important advantages are:
$\checkmark \quad$ Reduced cost
$\checkmark$ Reduced weight
$\checkmark \quad$ Increased accuracy
$\checkmark$ Compact size - notably with stroke length compared to conventional LVDTs
$\checkmark$ Simplified mechanical engineering, for example, eradication of seals, bearings, and bushes
$\checkmark$ Simplification of the electrical interface - typically a DC supply and absolute, digital signal

\section{CONCLUSIONS}

As a conclusion, if incremental and rotary encoders are compared, it is very hard to determine which is more suitable, since it depends on the requirements of the applications. For instance, if for less complex applications incremental rotary encoders are the best choice, but for complex and safetycritical applications absolute rotary encoders should be selected. One of the main advantages of using incremental ones is low maintenance cost and simple integration process. More detailed comparison for these two encoders are given in the table below. 
Table 2. Comparison of incremental and absolute encoders

\begin{tabular}{|c|c|c|c|}
\hline & \multicolumn{2}{|c|}{ Incremental } & Absolute \\
\hline & Single-channel & Quadrature & \\
\hline Complexity & \multicolumn{2}{|c|}{ Simpler } & More Complex \\
\hline Output & Speed, displacement & $\begin{array}{l}\text { Velocity and } \\
\text { direction }\end{array}$ & $\begin{array}{l}\text { Velocity and absolute } \\
\text { position }\end{array}$ \\
\hline $\begin{array}{l}\text { Needs homing } \\
\text { on startup? }\end{array}$ & \multicolumn{2}{|c|}{ Yes } & No \\
\hline Resolution & \multicolumn{2}{|c|}{ Up to $10 \mathrm{k}$ PPR (direct read) } & $\begin{array}{c}\text { Up to 22-bit (ST) / } 12- \\
\text { bit (MT) }\end{array}$ \\
\hline $\begin{array}{c}\text { Communication } \\
\text { via protocol? }\end{array}$ & No & No & Yes \\
\hline Cost & \multicolumn{2}{|c|}{ Generally lower } & Generally higher \\
\hline
\end{tabular}

An absolute encoder would allow operations to be seamlessly resumed when power returns. If certain type of protocols such as PROFINET, Ethernet, or J1939 are intended to use or after encoder stopped during power outage memorizing the last track of exact numerical value then absolute encoders are much more preferable.

Inductive rotary sensors differ the ones mentioned above in many aspects. Firstly, Inductive rotary sensors are contactless sensors and can be widely used in harsh environments. They are often the natural choice for safety-critical, safety-related, or high reliability applications such as in the aerospace, military, rail and heavy industrial sectors. Actually, inductive sensors are expensive because of the bulk of material used and the requirement for carefully wound coils. However, its physical robustness and quite high accuracy makes is reliable and widely-used. As mentioned above, new generation of inductive sensor has growing reputation not only in the traditional markets, but also in industrial, automotive, medical, utility, scientific, oil and gas sectors. It has more advantageous characteristics than the previous types of inductive rotary sensors. Using printed circuits on flexible or rigid substrates, instead of bulky spools of wire makes these sensors less expensive and more compact.

\section{RESULTS}

As a result, we can say that all the types of rotary sensors mentioned above have their advantages and disadvantages depending on the working conditions and environment. However, while the demand to these type of the sensors is getting to increase, the producers try to improve the capabilities and functions to be able to implement them in wide area of applications. Therefore, the new generation of rotary sensors are going to eliminate the disadvantages of the previous ones and they are used in many applications successfully nowadays.

\section{ACKNOWLEDGMENTS}

All authors would like to express their gratitude to the PostGraduate Program of Studies "Automation of Productions and services" of PUAS, for the financial support to undertake this research project

\section{REFERENCES}

[1] https://en.wikipedia.org/wiki/Sensor

[2] http://www.anaheimautomation.com/manuals/forms/enco der-guide.php\#sthash.IjKvhux1.f5Ba39Q6.dpbs

[3] http://howtomechatronics.com/tutorials/arduino/rotaryencoder-works-use-arduino/

[4] Joel Jacobs "Incremental \& Absolute Encoders: What's the Best Solution for Your Application?"

[5] http://www.ni.com/white-paper/14805/en/\#toc2

[6] https://www.ia.omron.com/support/guide/34/introduction .html

[7] https://en.wikipedia.org/wiki/Rotary_encoder

[8] http://www.dynapar.com/Technology/Encoder_Basics/In cremental_Encoder/

[9] http://blog.pepperl-fuchs.us/the-differences-betweenabsolute-and-incremental-rotary-encoders

[10] http://www.ifm.com/ifmmy/web/pinfo015_010_040.html

[11] Mark Howard of Zettlex, Precision in the Extreme, How Inductive Sensors work

[12] http://www.sensores-demedida.es/uploads/sensores_de_angulo_rvdt_dc.pdf

[13] https://www.azosensors.com/article.aspx?ArticleID=745

[14] Bill Schweber "Rotary Encoders Critical to Successful Motion-Control Implementation"

[15] David S. Nyce, "Position Sensors", pp.200-220, 17 JUNE 2016 